\title{
抗虎新药氨三肼 $(\mathrm{AT}-1902)$ 的研究
}

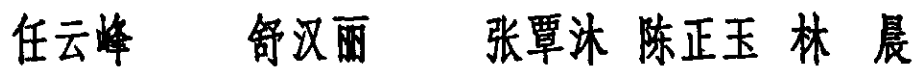 \\ (中国科学院上海药物研究所) (河南尛医学科学研究所)
}

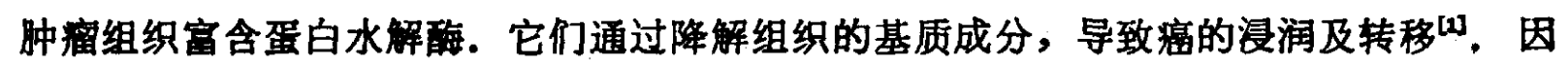
此, 抑制蛋白水解酶的活力, 有可能阻止癌的生长及播散.

许多蛋白水解酶如原胶原蛋白酶、组织蛋白酶、肽酶及纤溶酶等, 能被一些络合剂如乙二 胺四乙酸所抑制[2].

碳酸胼是一种能量代谢抑制剂. 它在磷酸烯醇丙酮酸羫激酶的水平上,千扰糖元异生，从 而对某些动物肿演有一定的抑制作用 ${ }^{[3]}$.

作者等在合成一系列酰貄衍生物的过程中, 合成了若干络合剂的酰㭌衍生物, 目的在于将 上述两种药理作用结合一起，以寻找有效抗癌药物. 结果发现, 宔三乙酰肼 (氨三訮, I, AT1902）及乙二胺四乙酰肼（AT-1903，II）对多种动物肿瘦有效,尤以氮三肼的疗效更好. 本 文简要报道虫三肤的化学及药理研究.
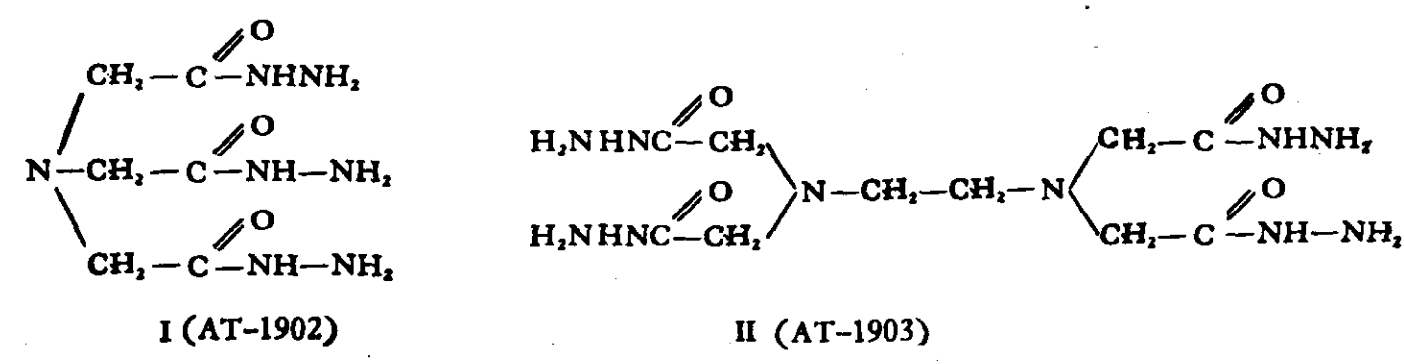

\section{一、化学研究}

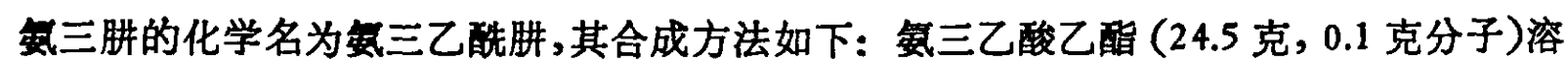
于无水乙 (100 毫开)中,加人水合肼 (85\%) (25 毫升, 约 0.4 克分子), 室温下放置, 不久即 析出油状物 ${ }^{[1]}$. 此物不易结晶, 放置数月后, 方旺结晶析出, 但若加人少量晶种, 则在 $1-2$ 天 内, 全部油状物即呈结晶析出. 过溏, 用无水乙醇洗, 得无色结晶 18.5 克(产率 $82.7 \%$ ), 熔点 $118-120^{\circ} \mathrm{C}$.

此物略具吸水性. 分析样品以稀乙醇重结晶.

元素分析: $\mathrm{C}_{6} \mathrm{H}_{15} \mathrm{~N}_{7} \mathrm{O}_{3}$ 分子量: 233.24

计算值\%：C 30.90； H 6.48； N 42.04.

实验值\%: C 31.23； H 6.40; N 42.22.

本文 1980 年 12 月 15 日收到. 


\section{二、药理研究}

1. 疗效试验每天腹腔注射氨三肼 200-250 毫克/公斤, 连续 5 天, 对大鼠 $\mathrm{W}-256$ 的 抑制率为 73-84\%，剂量减至 100 毫克/公斤，抗瘁作用明显减弱; 口服 200 毫克/公斤/天 $\times$ 5 , 对 W-256 的抑制率亦达 $77.6 \%$ 。腹腔注射氨三肼 250 毫克/公斤/天 $\times 10$, 对小鼠艾氏癌 (实体型)及肝癌 (实体型)的抑制率分别为 $69.5 \%$ 及 $55.1 \%$, 对脑瘦 $\mathrm{B}_{22}$ 亦有一定抑制作用, 但

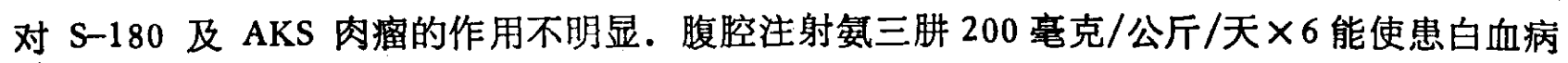
L-615 小鼠的生存期延长·127-150\%, 口服给药的疗效更好, L-615 小鼠的生存期延长达 $310 \%$, 且有部分动物长期存活. 腹腔注射氨三肼 200 毫克/公斤/天 $\times 6$, 对患腹水型 $\mathrm{W}-256$ 大鼠的存活期无明显影响, 但口服氨三肼 100 毫克/公斤/天 $\times 6$, 则能延长其存活期 $36.7 \%$ $(\mathrm{P}<0.01)$. 若腹腔注射硫酸肼 40 毫克/公斤/天 $\times 6$ 对大鼠 $\mathrm{W}-256$ 的抑制率为 $56.5 \%$, 而 腹腔注射氨三乙酸 40 毫克 $/$ 公斤 $/$ 天 $\times 7$ 对 W-256 无效; 氨三乙酸与硫酸胖合用, 抗痹作用亦 未见增加. 这说明氨三肼的抗瘤作用似与其自身的结构特点有关.

氨三肼对大鼠 W-256 的疗效与给药方案有一定从属关系. 在总剂量. (900 毫克/公斤) 相同的情况下, 分 6 次 (每天给药)、3 次 (每二天一次)、2 次 (每三天一次)及一次大剂量给药 的疗效分别为 $22.4 \% 、 52.8 \% 、 17.2 \%$ 及 $21.7 \%$. 这说明每二天给药一次的序效最好.

实验证明,维生素 $\mathrm{B}_{6}$ 可完全对抗氨三肼的抗痹作用,亦可对消其毒性 (体重下降的作用). 去篻吡略酫与氨三肼合用,则抗溜作用相加.

在组织培养液中, 氨三肼在 200-500 微克/言升的浓度下, 对艾氏腹水癌细胞无明显的细 胞毒作用. 考虑到腹腔注射氨三肼对小鼠 S-180 (腹水型)、艾氏腹水癌及大鼠 W-256（腹 水型)均无效, 但口服给药却能延长患腹水型 W-256 大鼠的生命, 这提示氨三肼可能在体内 需先转化为有效的代谢产物方能显示抗㿔作用.

溶血素反应试验及肿瘤相伴免疫试验的结果表明, 氨三肼对两者均无明显影响, 但在 200-500 毫克/公斤剂量下, 对移植物抗宿主试验可以明显提高脾指数.

2. 每性试验 氨三册对小鼠的急性 $L D_{500}$ 腹腔注射为 1736 (1437-2035) 毫克/公 斤, 口服给药为 $1343(1128-1559)$ 毫克/公斤. 给一次维生素 $B_{6}$ 或去氧吡哆醛对氨三肤的 $L D_{90}$ 无明显影响.

对家兔的亚急性毒性试验结果表明, 每天静脉注射氨三肼 12.5 毫克/公斤, 连给 7 天, 除 体重稍有下降外, 余者无明显反应, 以同样的剂量口服给药, 则有 $2 / 4$ 家兔死亡. P检可见胃 肠粘膜充血及出血点, 但肝、肾、肺、心等组织均未见明显异常. 若改为隔天给药, 即使剂量增 加一倍, 即 25 毫克 $/$ 公斤 $/ 2$ 天 $\times 7$, 则不论静脉或口服给药, 均无家兔死亡.

对六条犬的亚急性毒性试验结果表明: 静脉注射氨三肼 10 毫克/公斤/天 $\times 7$, 二犬中有 一犬进食量减少, 体重下降, 并出现呕吐; 剂量增至 15 毫克/公斤/天 $\times 7$, 二犬中有一犬出现 呕吐及便血, 体重明显下降, 血红蛋白轻度下降, 停药二周后恢复; 静脉注射 20 毫克/公斤/ 天 $\times 7$, 二犬均见剧烈呕吐、便血、不食, 停药后给予补液才逐渐诙复.

给犬口服氨三肼 10 毫克/公斤/天 $\times 7$, 除出现食量减少、呕吐及体重下降外, 无其他明显 毒性反应; 剂量增至 15 毫克/公斤/天 $\times 7$, 给药二次后, 二犬均不食, 呕吐、便血, 其中一犬于 用药 5 天后死亡; 户检发现胃肠粘膜充血, 有出血点; 组织学检查肝、肾、肺、心等脏器均无明显 
病理学变化。

不论口服或静注,亦不论犬或家兔,在毒性剂量下,氨三肼对骨能功能均无明显影响.

管三肤已进行第 I 期临床试验.初步结果显示,每天口服 180 毫克 (分三次服),连续 1-2 月,除个别病人显示轻度消化道反应外，对肝、肾、心、肺、骨鲔功能均无明显影响.

\section{$\Rightarrow \quad \Rightarrow \quad$ 文}

[1] Keiditsch, E. \&trauch, L., in Chemistry and Moleoular Biology of Intracellular Matrix (Ed. Balas, E. A.), Academic Press, London, Now York, 8(1970), 1671.

[2] Boggust, W. A., in Advances in Tumour Prevention, Detection \& Characterization (Ed. Maltoni, C.), American Elsevier Publishing Co. Inc., New York, 1976, vol. 3.

[ 3 ] Gold, J., Oncology, 25(1971), 66; $27(1973), 69$.

[4] Curtius, T. \& Hoffmann, O., J. Prak. Chem. (II), 96(1917), 202-235. 